This item was submitted to Loughborough's Research Repository by the author.

Items in Figshare are protected by copyright, with all rights reserved, unless otherwise indicated.

\title{
Investigating the effects of beat and deictic gestures of a lecturer in educational videos
}

PLEASE CITE THE PUBLISHED VERSION

https://doi.org/10.1016/j.compedu.2020.103955

PUBLISHER

Elsevier

VERSION

AM (Accepted Manuscript)

\section{PUBLISHER STATEMENT}

This paper was accepted for publication in the journal Computers \& Education and the definitive published version is available at https://doi.org/10.1016/j.compedu.2020.103955.

LICENCE

CC BY-NC-ND 4.0

\section{REPOSITORY RECORD}

Beege, Maik, Manuel Ninaus, Sascha Schneider, Steve Nebel, Julia Schlemmel, Jasmin Weidenmüller, Korbinian Moeller, and Günter Daniel Rey. 2020. "Investigating the Effects of Beat and Deictic Gestures of a Lecturer in Educational Videos”. Loughborough University. https://hdl.handle.net/2134/12630500.v1. 


\title{
Investigating the effects of beat and deictic gestures of a lecturer in educational videos
}

\author{
Maik Beege $^{1}$, Manuel Ninaus ${ }^{2,3}$, Sascha Schneider ${ }^{1}$, Steve Nebel ${ }^{1}$, Julia Schlemmel ${ }^{1}$, \\ Jasmin Weidenmüller ${ }^{1}$, Korbinian Moeller²,3,4, Günter Daniel Rey ${ }^{1}$
}

\author{
${ }^{1}$ Psychology of learning with digital media, Faculty of Humanities, Chemnitz University \\ of Technology, Germany \\ ${ }^{2}$ Leibniz-Institut für Wissensmedien, Tuebingen, Germany \\ ${ }^{3}$ LEAD Graduate School and Research Network, University of Tübingen, Tübingen, \\ Germany \\ ${ }^{4}$ Department of Psychology, University of Tübingen, Tübingen, Germany
}

Corresponding author: Maik Beege

E-Mail: maik.beege@phil.tu-chemnitz.de

Phone: 0049371 / $531-31758$

Psychology of learning with digital media

Faculty of Humanities

Chemnitz University of Technology

Straße der Nationen 12

09111 Chemnitz, Germany 
- Deictic gestures but not beat gestures enhance learning outcomes

- Signaling visual material through deictic gestures enhance attention and social presence

- Agent-persona perception is influenced by gestures of a lecturer

- Evidences for the signaling but not for the embodiment principle were found 


\title{
Investigating the effects of beat gestures and deictic gestures of a lecturer in educational videos
}

\begin{abstract}
Lecturers in educational videos often use gestures to emphasize what has been said or highlight learning relevant information, which is visible on the screen. However, differences in types of lecturer gestures, such as rhythmic (beat) gestures and signaling (deictic) gestures, have not been investigated thoroughly yet concerning human lecturers in educational videos. In two experiments $\left(N_{1}=108 ; N_{2}=121\right)$, participants received an educational video about weather phenomena (Experiment 1) or the industrial revolution (Experiment 2). Videos were manipulated in terms of the type of lecturer's gestures in the video (beat gestures vs. deictic gestures vs. no gestures). Learning outcomes, mental load and effort, parasocial interaction, social presence, affective rating, and agent-persona perception (Experiment 2) were measured. Results indicated a significant effect of gestures on retention performance in both experiments. In line with the signaling principle, deictic gestures enhanced learning outcomes. In contrast, beat gestures did not foster learning in comparison with a video without gestures. These results are interpreted considering lower mental load, higher social presence, and parasocial interaction in the signaling condition. In particular, attention towards the lecturer was significantly enhanced in the condition with deictic gestures in both experiments.
\end{abstract}

Keywords: educational videos; signaling; deictic gestures; social cues; beat gestures. 


\section{Introduction}

Gestures are considered essential aspects of human communication (McNeill, 1992). They are not only natural movements but also habitually used in conversations. Interestingly, gesturing even occurs in situations in which no dialog partner is visible, for example, during a telephone call. Moreover, even people who are blind from birth use gestures in conversations although they have never seen somebody gesturing (Iverson \& Goldin-Meadow, 1998).

Supporting the oral communication in daily routine and adding a visual component to spoken language is a unique ability of gestures. In particular, gesture and language play a decisive role in the context of multimedia presentations and are often intertwined. Because lecturer-based instructional videos steadily gain popularity in scientific investigations (e.g., Kizilcec, Bailenson, \& Gomez, 2015), researchers, who focus on learning with educational videos, have increased interest in gestures. Scientific studies regarding gestures of actual human lecturers showed that gestures interact with the spoken message and thus, gestures are part of the cognitive representation of the lecture (Cassell, McNeill, \& McCullough, 1999). Thus, nonverbal communication from a human lecturer is important for learning and can enhance learning outcomes from recipients (Novack \& Goldin-Meadow, 2015; Ouwehand, van Gog, \& Paas, 2015; Congdon, Novack, Brooks, Hermani-Lopez, O'Keefe, \& Goldin-Meadow, 2017). Most research about the use of gestures in learning environments was carried out with digital agents and not with actual humans. These studies showed that implementing gestures can improve learning as well (e.g., Atkinson, 2002, de Koning \& Tabbers, 2013; Li, Wang, Mayer, \& Liu, 2019).

However, in contrast to these beneficial effects, gestures might also attract the attention of the learner and consequently distract her/him from the actual learning task (e.g., seductive detail effect; Harp \& Mayer, 1998; Sundararajan \& Adesope, 2020). The social cues of gestures might 
be seen as extraneous stimuli, which impede learning by leading to cognitive overload. As such, one might hypothesize that in educational videos, where additional pictorial material is presented, learning might be fostered when there are no gestures. These contrasting views clarify the need to investigate how and under which the gestures' conditions the use in educational videos foster learning.

\subsection{Defining and Categorizing Gestures}

Movements of hands and arms during a conversational act are an omnipresent function of human-to-human interaction (Cook, Duffy, \& Fenn, 2013). Roth (2001) identified gestures as the dominant element of human communication across cultures. Following the definition by McNeill (1992), gestures are spontaneous movements that occur synchronized with language. According to McNeill (1992), gestures can illustrate pictures and thoughts that are difficult or even impossible to express through language alone. In the same context, Goldin-Meadow (2011) emphasizes that gestures convey information beyond what has been said because gestures provide insights into a person’s thoughts and are of central importance for learning (Novack \& GoldinMeadow, 2015) as well as for other cognitive processes (Stieff, Lira \& Scopelitis, 2016).

McNeill (1992) established one of the most prominent taxonomies of gestures. Accordingly, gestures can be distinguished into four types: (i) beats, (ii) deictic gestures, (iii) iconic gestures, and (iv) metaphorical gestures. (i) Beats summarize all hand movements that occur in the rhythm of the language. They are non-pictorial gestures, which emphasize verbal information. Even if "beats are the most insignificant looking; but appearances are deceptive, for beats are among the most revealing of gestures, uncovering the speaker's construction of the narrative discourse" (Cassell and McNeill, 1991; S. 384). Examples are gestures of raising and lowering hands as well as beating or tapping movements of the hands. McNeill defines (ii) deictic gestures as concrete or 
abstract pointing movements, which are always context-sensitive. Gestures, which illustrate what is said just in time and in a pictorial way are called (iii) iconic gestures. They demonstrate a close semantic connection between speech and concurrently performed hand movements. The last category of gestures, so-called (iv) metaphoric gestures, are very similar to iconic gestures. However, the illustrated content does not refer to something physically present, but to a more abstract idea. As such metaphoric gestures aim at illustrating an invisible image by means of abstraction.

Research regarding human lectures often investigated only deictic gestures (e.g., Novack \& Goldin-Meadow, 2015) or rhythmic gestures (Cassell et al., 1999) and postulated benefits of gesture implementation. Nevertheless, studies in the context of learning that investigated only rhythmic gestures of human lecturers are rare. Concerning gestures of virtual agents, Cook, Friedman, Duggan, Cui, and Popescu (2017) postulated that learning with animations should be fostered when an implemented pedagogical agent used deictic and rhythmic gestures. However, the pedagogical agent performed both types of gestures. Accordingly, it was not possible to distinguish which type of gesture ultimately fostered learning. With reference to the taxonomy of gestures by McNeill (1992), the current study investigates the impact of the two mentioned types of gestures. In this context, rhythmic (beat) gestures might act as additional social cues which draw the attention of the learner to the lecturer in an educational video and mobilize cognitive resources which might foster learning (Mayer, 2014). On the other hand, deictic gestures may cue important parts of pictorial material within the video. This signaling of important visual information might foster learning in turn (Schneider, Beege, Nebel, \& Rey, 2018a). 


\subsection{Gestures as Social Cues}

Computer or video-based instruction can be interpreted as a social event (social agency theory; Reeves \& Nass, 1996; CASA paradigm; Nass, Steuer \& Tauber, 1994). Social cues in an instructional media environment (e.g., voice, eye contact) prime a social activation schema while social processes of human-to-human communication are triggered (Grice, 1975). According to Mayer (2001), cognitive processes like selecting, organizing and integrating relevant verbal as well as visual information into a coherent mental representation are enhanced by social cues because learners do not only interpret educational videos as a piece of information but also as a situation of social communication. Thus, relevant cognitive processes of selecting and organizing information are engaged in order to understand the sense of the provided information. Furthermore, Park (2015) showed that social cues in multimedia learning environments lead to lower mental load with a medium effect size $\left(\eta^{2}=.08\right)$. In fact, learners seemed to work harder and tended to invest more cognitive effort in learning when social cues are implemented in learning environments.

According to the embodiment principle as introduced by Mayer (2014), beat gestures nicely fit in the category of “social cues”. In particular, Mayer (2014) stated that “...people learn more deeply when on-screen agents display humanlike gesturing, movement, eye-contact and facial expressions.” (p. 345). In this vein, beat gestures draw the attention of the learner directly to the lecturer (and therefore, directly to the speech; cf. Wakefield, Novak, Congdon, Franconeri, Goldin-Meadow, 2018) in the video and possibly foster learning processes. Numerous studies investigated gestures as an embodied cue and observed beneficial effects on learning, in particular considering retention (Brooks \& Goldin-Meadow, 2016; Cook, Mitchell, \& Goldin-Meadow, 2008). Importantly, learners did not only benefit from gesturing themselves but also form 
observing gestures (e.g., Wang, Li, Mayer, \& Liu, 2017; Ping, Goldin-Meadow, \& Beilock, 2014) - in line with the embodiment principle proposed by Mayer (2014, in contrast to theories of embodied learning as representational grounding of mental concepts through physical interactions with the environment, e.g., Barsalou, 2010; Glenberg, 2010, see also Skulmowski \& Rey, 2018). In this sense, watching somebody's gestures is assumed to activate the motor system of the observer as an additional embodied cue to the learning content (Wang, Li, Mayer, \& Liu, 2017; Ping, Goldin-Meadow, \& Beilock, 2014). This could be shown in studies with human lecturers performing gestures (e.g., Ping, Goldin-Meadow, \& Beilock, 2014; Wakefield et al., 2018). Observing a lecturer with beat gestures in an educational video creates the sense of social presence (i.e., awareness of another social entity during a communication process; Blascovich \& Bailenson, 2011; Lee \& Nass, 2003) and leads to a social response and affective change (Um, Plass, Hayward, \& Homer, 2012). Gestures from human lecturers are processed and stored in memory when they support or even contradict the verbal information (Cassell et al., 1999) and foster recall processes. Interestingly, studies utilizing pedagogical agents came to the same conclusion (e.g., Wang, Li, Mayer, \& Liu, 2017). Salem, Kopp, Wachsmuth, Rohlfing, and Joublin (2012) pointed out that even robots are evaluated more positively when hand and arm gestures are displayed. Moreover, Davis and Vincent (2019) postulated that beat gestures shown by pedagogical agents helped learners to comprehend more during language acquisition because beat gestures might result in enhanced parasocial processes (Hartmann, Schramm \& Klimmt, 2004; Horton \& Wohl, 1956). Parasocial interaction (PSI) may be defined as the conversational give and take between a persona and a recipient that has multiple influences on the recipients and, therefore, potential learners (Beege, Schneider, Nebel, \& Rey, 2017; Horton \& Wohl, 1956; Gola, Richards, Lauricella, \& Calvert, 2013; Hartmann et al., 2004). The term “para”social was introduced, since 
the communication is unidirectional and traditionally, no back channel from the recipient to the media persona is available. An enhanced PSI is associated with perceptive-cognitive processes such as attention, understanding, and evaluation and was shown to foster learning with a large effect size $\left(\eta_{\mathrm{p}}^{2}=.14\right.$; Beege, et al., 2017).

In sum, beat gestures in an educational video might enhance parasocial processes (Hartmann et al., 2004), perceived social presence (Blascovich \& Bailenson, 2011), and learning relevant processes like selecting and organizing information. According to the social agency theory, beat gestures might foster learning regardless of whether they are observed in a classroom or media environment - a claim supported by recent empirical evidence with human lecturers (e.g., Cassell et al., 1999, Koumoutsakis, Church, Alibali, Singer, \& Ayman-Nolley, 2016) as well as pedagogical agents (e.g., Wang et al., 2017).

\subsection{Gestures as Signaling Cues}

Besides beat gestures, which draw the attention of the learner to the lecturer, deictic or signaling gestures can draw the attention of the learner to relevant pictorial parts of the educational video (McNeill, 1992). Therefore, learning relevant influences of deictic gestures can be explained by considering the signaling principle (for reviews, see: Schneider, et al., 2018a). The signaling principle or the cueing principle postulates that learning is fostered when instructional materials include visual or auditory cues that highlight relevant elements or the organization of the material. As such, signaling may reduce learning-irrelevant processing (e.g., Amadieu, Mariné, \& Laimay, 2011) and therefore overall mental load. In particular, in instructional materials, which are unknown to the learners and without a guiding teacher, learners might not intuitively recognize which pieces of information are learning-relevant and which might be irrelevant. 
Furthermore, educational videos usually show visual information beyond learning-relevant information (e.g., pictures of the explained objects or maps). The lecturer can use deictic gestures to specify which piece of information is necessary for understanding and to direct attention to this information. This approach is used to improve video instructions with actual humans (e.g., Koumoutsakis et al., 2016; Pi, Xu, Liu, \& Yang, 2020). Without signaling through gestures, learners might miss relevant pieces of information because they need to split their attention between the lecturer and visual information displayed in the educational video. A current metaanalysis compellingly demonstrated detrimental effects of learning material in which learners have to split their attention between different sources of information (Schroeder \& Cenkci, 2018). Support is provided by studies using pedagogical agents. Deictic gestures shown by a digital agent had a positive impact on learning (e.g., Atkinson, 2002; Dunsworth \& Atkinson, 2007). So far, studies with digital agents have gone much further into detail and substantiated the discussed findings. Gestures from digital agents seemed to be beneficial for learning when the agent pointed directly to the relevant information (Craig, Twyford, Irigoyen, \& Zipp, 2015) and not just roughly in the direction (Craig, Gholson, \& Driscoll, 2002). Furthermore, gestures should be implemented exactly at the moment or slightly after the information is presented verbally (temporal contiguity; Twyford \& Craig, 2013) and supported by facial expressions (Baylor \& Kim, 2009).

Taken together, signaling or deictic gestures highlight relevant visual information which decreases overall mental load during learning, which, in turn, can foster learning processes and enhance learning outcomes (Boucheix, Lowe, Putri, \& Groff, 2013; Lin \& Atkinson, 2011) with human and digital lecturers (Cook et al., 2013; Davis, 2018; Dunsworth \& Atkinson, 2007). 


\section{Experiment 1}

The current study aimed at comparing the effect of beat and deictic gestures in educational videos. This is especially relevant because current research regarding the influence of gestures on learning rarely differentiated between these two types of gestures experimentally (e.g., Cook et al, 2017). Only one recent study could be identified which differentiated between different types of gestures (Li, Wang, Mayer, \& Liu, 2019) and this study investigated virtual agents and not actual humans. According to the authors, only deictic gestures improved learning from pedagogical agents. Nevertheless, the influence of different kinds of gestures from humans in instructional videos are rarely investigated. Especially, investigations in terms of beat gestures in learning scenarios are rare and the most detailed investigations with regard to deictic gestures were carried out with pedagogical agents. Furthermore, most studies focused on the investigation of learning outcomes (e.g., Craig, Twyford, Irigoyen, \& Zipp, 2015) and did not measure several subjective variables which can provide information why gestures might be beneficial (i.e., parasocial interaction).

By manipulating educational videos in terms of the lecturer's gestures (no gestures $\times$ beat gestures which should support the rhythm of the speech $\times$ deictic gestures which highlighted relevant areas of the additional pictorial information), the current investigation aims to provide three key implications: (i) to evaluate whether the embodiment principle (e.g., Mayer, 2014) and the signaling principle (e.g., Schneider, et al., 2018a) can be supported by gestures in an educational video; (ii) to outline of the effectiveness of two different types of gestures used by actual human lecturers in educational videos and (iii) to provide additional information on the underlying processes during learning from lecturers using different gestures. 
According to the literature discussed above, beat gestures provide additional social cues, which might lead to enhanced learning relevant processes (Mayer, 2014). They cause enhanced attention of the learner on the lecturer and the speech itself, because these social cues prime the activation of a social response. As mentioned before, implementing social cues in learning environments can foster learning (Beege, et al., 2017). In contrast, deictic gestures guide the attention of the learner to learning relevant parts of the educational video and therefore, away from the lecturer. According to the signaling principle, deictic gestures should also foster learning (cf. Schneider, et al., 2018a; for a meta-analysis). Since both kinds of gestures can be associated with enhanced learning outcomes, the current study aimed to differentiate possible learning relevant effects and to provide specific design recommendations. The following research question and two hypotheses were pursued for the first experiment:

H1: Learners who watch an educational video with a lecturer showing beat gestures achieve higher learning outcomes than learners who watch an educational video with a lecturer showing no gestures.

H2: Learners who watch an educational video with a lecturer showing deictic gestures achieve higher learning outcomes than learners who watch an educational video with a lecturer showing no gestures.

RQ1: Do students who watch an educational video with a lecturer showing deictic gestures or beat gestures differ regarding learning scores?

To gain deeper insights into the effects of gestures, additional variables were investigated. The discussed literature showed that cognitive processes (e.g., cognitive load; Amadieu, et al., 2011; Park, 2015) and affective variables (e.g., affective rating of the material; Um et al., 2012) are influenced by social cues in multimedia learning environments. Yet, according to the CASA paradigm and the social agency theory, social variables should be in the focus when recording data of underlying processes. Because learning relevant cognitive processes are fostered through (para)social communication processes (Grice, 1975; Mayer, 2001), the strength and nature of the 
processes could provide more precise information on how learning works in parasocial digital learning environments. Accordingly, social processes (parasocial interaction; Beege et al., 2017; social presence; Blascovich \& Bailenson, 2011) were measured and investigated as well.

\subsection{Method}

Participants \& Design. Overall, studies regarding influences of social cues and signaling gestures showed medium to large effect sizes (e.g., Schneider et al., 2018a for a meta-analysis). An a-priori power analysis with G*Power (Faul, Erdfelder, Lang, \& Buchner, 2007) considering a large effect size $(f=.40, \alpha=.05)$, outlined that 66 participants should at least be acquired for the current design to observe the effect with sufficient statistical power (i.e., $1-\beta=.80$ ). Because observed effect sizes in previous research were medium to large, 108 university students (85.2\% female; age: $M=22.33, S D=3.99$ ) from the $\mathrm{XXX}$ (anonymized because of peer review) participated in this experiment. No participant had to be excluded. Participants were randomly assigned to one group of a one-factorial between-participant design with three factor levels (no gestures vs. beat gestures vs. deictic gestures) by an online randomization systemThe participants studied media communications (76\%), general psychology (4.8\%), teacher education (7.7\%) and other fields (11.5\%). Thirty-six students were assigned to the condition with no gestures, 33 students were assigned to the condition with beat gestures and 39 students to the condition with deictic gestures. There were no significant differences between the three groups in terms of age, or prior knowledge, $F[2,103]=[0.27,1.76] ; p=[.18, .77]$ as well as gender, familiarity with the lecturer, or field of study $\chi^{2}=[0.44,5.76] ; p=[.45, .80]$. In consequence, there was no need for including additional covariates in the analyses.

Materials. Three educational videos were filmed in front of a blue screen in a TV-studio to implement the three experimental conditions. Within these videos, a pre-trained male lecturer held 
a presentation on facts about weather phenomena. The lecture contained information about cause, appearance, and course of trade winds, tornados, and hurricanes. The content was chosen because it was considered that prior knowledge of participants was low and additional graphics could be designed to provide further explanations in the video. This is especially relevant because educational videos like Massive Open Online Courses (MOOCs) often contain accompanying visual material (PowerPoint presentation, etc.) strengthening the external validity of our experiment. Overall, the experimental videos lasted about 9:14 minutes.

Lecturer. The lecturer was presented in the right half of the screen (see Figure 1). He never turned away and constantly looked at a teleprompter, which stood slightly to the right of the camera. Therefore, the eye gaze of the lecturer was kept constant across experimental conditions. In the condition without gestures (control condition), the hands of the lecturer were not visible. The lecturer held his oral presentation without moving.

In the condition with rhythmic gestures, the lecturer used beat gestures constantly to rhythmically support his oral presentation. These gestures were nondescript and were implemented to provide additional social cues. Therefore, special care was taken to ensure that the used gestures did not convey unintentional semantic content.

In the condition with deictic gestures, the lecturer intentionally pointed on specific areas of the additional material, which was displayed in relevant parts of the video. Furthermore, the lecturer used gestures to illustrate proportions und underline relevant parts of the presentation. Because the lecturer was pre-trained, his speech rate and therefore, the length of the videos of the condition was kept almost constant. 
Additional pictorial material. After recording the videos, a neutral blue background was placed over the blue screen. The oral presentation was visually supported by pictorial material, which was shown at the left side of the screen. Illustrations were updated constantly and showed relevant processes of the weather phenomena. In the deictic condition, illustrations were placed in a way that they matched with the deictic gestures of the lecturer. To avoid copyright issues and to get illustrative material that explicitly fitted to our presentation, all illustrations were custom-made. Examples of the material regarding the emergence of a tornado are displayed in Figure 2.

\section{Insert Figure 2 about here}

Measures. The coefficient McDonald's omega ( $\omega$; McDonald, 1999; McNeish, 2017) was chosen in order to calculate the reliability for all measures. For interpretation the conventions for the interpretations from Cronbach's $\alpha$ were used. In appendix A, all items of all scales are displayed.

Mental Load and Mental Effort. Mental load and mental effort were measured with a questionnaire from Krell (2015). Six items assessed mental load (ML; $\omega=$.95; e.g., “The tasks were challenging”) and another six items assessed mental effort (ME; $\omega=.92$; e.g., "I have made an effort at the processing of the tasks”). Items were rated on a 7-point Likert scale ranging from "not at all” to “totally”.

Affective Rating. To assess affective rating of the videos, a scale from Linnenbrink-Garcia, Durik, Conley, Barron, Tauer, Karabenick, and Harackiewicz (2010) was implemented. The scale consisted of four items which were rated on a 7-point Likert scale ( $\omega=.96$; e.g., "I enjoyed watching the educational video”) ranging from “does not indicate the case at all” to "completely indicates the case”. 
Social Presence. To measure social presence, a scale from Bailenson, Aharoni, Beal, Guadagno, Dimov, and Blascovich (2004) was used. Five items measured the construct on a 7point Likert scale ( $\omega=.64$; e.g., "The lecturer seemed feeling and alive") ranging from "does not indicate the case at all" to "completely indicates the case".

Parasocial Interaction. Parasocial interaction was measured with specially selected items from the PSI process scales (Schramm \& Hartmann, 2008). Eight items were chosen $(\omega=.67)$ which measured the four sub-facets which we considered highly relevant for learning. The first sub-facet was the accuracy of processing lecturer-related information (one item). Learners were asked how well they still remember what the lecturer talked about. The second sub-facet was attention towards the lecturer (two items). Learners were asked how much attention they paid to the lecturer. The third sub facet measured the logical understanding of the behavior of the persona (two items). Learners should assess how thoroughly they thought about what was said by the lecturer. The fourth sub-facet dealt with the evaluation of the persona (three items). Learners were asked whether they found the statements of the lecturer good or bad and whether they formed their own opinion on the information. Students had to rate these items on a 7-point Likert scale ranging from "does not indicate the case at all” to "completely indicates the case".

Prior Knowledge. Prior knowledge was assessed by three self-report questions. Students had to answer the questions: "What do you already know about trade winds/tornados/hurricanes?" When students reported detailed information about the weather phenomenon, they got one point. In case students reported trivial or minor information, they achieved 0.5 points. When students reported no or incorrect information, they got 0 points. Therefore, students were able to achieve three points regarding prior knowledge. The inter-rater reliability was perfect (ICC = 1; Shrout \& Fleiss, 1979) 
Retention and Transfer. Retention $(\omega=.55)$ and transfer tests $(\omega=.71)$ were implemented in order to adequately assess learning performance. Retention can be defined as remembering or reproducing information which were presented in the instructional videos and transfer refers to applying the knowledge in order to solve novel problems, which were not explicitly presented in the learning material (Mayer, 2014). The rather low reliabilities of the retention test can be explained by considering the item construction. The retention and transfer scale aimed to assess different sub-topics (different weather phenomena, the emergence of specific phenomena, geographical appearance, etc.). The items asked for information of various difficulty and complexity. Thus, both scales were deliberately created multidimensional to generate a broad variance in the responses. Detailed applicable knowledge in one domain does not necessarily mean that the participants could apply their knowledge correctly to other items. Consequently, reliability, as measured with McDonalds $\omega$, was restricted.

Retention was measured with 16 single choice questions (e.g., "What is the name of trade winds in the northern hemisphere?”). The single choice questions consisted of four possible answers and only one answer was correct per question. Participant gained 1 point when they marked the correct solution probe. Therefore, participants could gain a maximum of 16 points in the retention questionnaire.

Transfer was measured with two single-choice and six open questions (e.g., "Hurricanes mostly occur from July till November. Why is this the case?”). The same question format as for retention was used to gather the transfer scores with single choice questions. The open questions had to be answered with a short sentence or bullet points. When the question was answered correctly, students could gain 1 point. In case the answer was partially correct, 0.5 points were awarded. Overall, students were able to gain a maximum of eight points in the transfer 
questionnaire. Raters were trained with a list of possible answers to ensure objective rating. Interrater reliability of two pre-trained reviewers was perfect $($ ICC $=1)$.

Procedure. The experiment took place in a computer lab in XXX and lasted 45 minutes. A maximum of four students simultaneously participated in this study. In order to guarantee that students work for themselves, partition walls were installed between workstations. At first, students received an URL to the electronic questionnaire, which consisted of the implicit automatic randomization and the prior knowledge test. Afterwards, students received the video either without gestures, with beat gestures or with deictic gestures. Students could not pause the video or repeat specific parts. Students were given headphones to individually adjust sound and watch the video undisturbedly. After finishing the video, students completed a set of questionnaires in the following order: parasocial interaction; affective rating; retention; transfer; mental load and mental effort. In the end, demographic data (age, sex and field of study) were assessed.

\subsection{Results}

To evaluate differences between the three experimental groups, (multivariate) analyses of variance, (M)ANOVAs were conducted. The experimental factor "gesture instruction" (control vs. beat gestures vs. deictic gestures; in the following referred to as "gesture instruction") was used as independent variable. (M)ANOVA preconditions were examined and only reported when significantly violated. Furthermore, effect sizes for all effects are only reported when significant effects $(p<.05)$ were reached. Sidak corrected post-hoc analyses were only reported for significant results. Descriptive results for all dependent variables are displayed in Table 1.

Insert Table 1 around here. 
Affective Rating. An ANOVA with affective rating as the dependent variable was conducted. No significant effect was found for the between-subject factor gesture instruction; $F(2,105)=$ $1.52, p=.22$.

Social Presence. An ANOVA with social presence as dependent variable revealed a significant effect with a medium effect size; $F(2,105)=6.45, p=.002, \eta_{\mathrm{p}}{ }^{2}=.11$. Sidak-corrected post-hoc analyses revealed that students in the deictic condition reported a higher score of social presence than students in the control condition $\left(M_{\text {diff }}=0.62 ; p=.002\right)$.

Parasocial Interaction. To evaluate PSI effects, a MANOVA was conducted with PSI subfacets (accuracy of persona-related information, attention, logical understanding, evaluation) as dependent variables. A significant effect with a medium effect size was observed for gesture instruction; Wilk's $\Lambda=0.82 ; F(8,204)=2.67, p=.01, \eta_{\mathrm{p}}^{2}=.10$. Univariate ANOVAs revealed a significant effect with a large effect size for the facet attention towards the persona; $F(2,105)=$ 8.38, $p<.001, \eta_{\mathrm{p}}^{2}=.14$. Sidak-corrected post-hoc analyses revealed that students in the deictic condition reported a higher attention than students in the control condition $\left(M_{\mathrm{diff}}=1.35 ; p<.001\right)$. With respect to the Sidak-procedure, none of the other pairwise comparisons reached significance. Nevertheless, there were descriptive tendencies that students in the beat condition reported higher attention than students in the control condition ( $\left.M_{\text {diff }}=0.76 ; p=.09\right)$.

Mental Load and Mental Effort. A MANOVA was conducted with mental load (ML) and mental effort (ME) as dependent variables. A significant effect with a small to medium effect size was observed for the between-participant factor gesture instruction; Wilk's $\Lambda=0.90 ; F(4,208)=$ 2.73, $p=.03, \eta_{\mathrm{p}}^{2}=.05$. Univariate ANOVAs revealed a significant effect for ME; $F(2,105)=$ 3.72, $p=.03, \eta_{\mathrm{p}}^{2}=.07$, but not ML; $F(2,105)=1.82, p=.17$. 
Regarding ME, Sidak-corrected post-hoc analyses revealed that students in the beat condition reported lower ME than students in the control condition $\left(M_{\text {diff }}=0.43 ; p=.04\right)$. Additionally, there was a descriptive tendency that students in the beat condition reported lower ME than students in the deictic condition $\left(M_{\text {diff }}=0.38 ; p=.08\right)$.

\section{Learning Outcomes.}

A MANOVA was conducted with retention and transfer as dependent variables. There was a significant main effect with a medium effect size for gesture instruction; Wilk's $\Lambda=0.88 ; F(4$, $208)=3.38, p=.01, \eta_{\mathrm{p}}^{2}=.06$. Univariate ANOVAs revealed a significant effect with a medium effect size for retention; $F(2,105)=5.74, p=.004, \eta_{\mathrm{p}}{ }^{2}=.10$. Sidak-corrected post-hoc analyses revealed that students in the deictic condition achieved higher retention scores than students in the control condition $\left(M_{\mathrm{diff}}=1.33 ; p=.01\right)$ and in the beat condition $\left(M_{\mathrm{diff}}=1.27 ; p=.02\right)$. No significant effect was found for transfer; $F(2,105)=2.06, p=.13$. Descriptively, students in the deictic condition achieved the highest transfer score.

\subsection{Discussion and Limitations}

Experiment 1 aimed at comparing effects of beat and deictic gestures of a lecturer in an educational video in comparison of a control group with no gestures. Results demonstrated that deictic gestures enhanced retention performance but not transfer performance. Therefore, H2 can be supported partially. In contrast, beat gestures did not significantly foster learning outcomes. Thus, H1 was not supported. Regarding the research question, the current results showed that the deictic condition outperformed the beat condition in terms of retention.

As regards the missing effects of social presence, parasocial interaction, and learning results for the beat gesture condition, the embodiment principle was not supported generally. A possible explanation might be that additional illustrative material was included in the video. Inserts of these 
illustrations might have attracted the attention of the learners in regular intervals. Thus, the gesticulating lecturer may not have been the focus of attention of the learner and therefore the social response of the learner did not differ from the control condition. This assumption is substantiated by considering the social variables, which were measured in Experiment 1. Beat gestures did not improve subjective social presence and only descriptively improved attentionrelated parasocial processes. Therefore, possible benefits of social cues (e.g., Schneider et al., 2018) on learning may not have been triggered. However, for this reason, they were not distracting either and learning inhibiting effects (i.e., seductive detail effect; Harp \& Mayer, 1998; Sundararajan \& Adesope, 2020) did not occur. Overall, the findings from Cassell and colleagues (1999) cannot be supported in general. It seemed that rather unspecific beat gestures are not beneficial for schema construction if they do not interact with the additional material and control the attention of the learner carefully.

The implementation of deictic gestures in educational videos significantly enhanced social presence and attention towards the lecturer. Thus, the benefits of embodiment of a social entity in learning environments may depend on how the embodiment is implemented in the educational video and how well movements are interwoven with the learning content. Because the lecturer interacts with the additional illustrative material through deictic gestures, both the lecturer and the illustrations are always in the center of attention of the learner and - in line with parasocial theories - social information was processed more deeply (Hartmann et al., 2004, Klimmt et al., 2006). Moreover, because learners did not have to split their attention between the lecturer and the additional visual material. All these discussed findings might explain the obtained signaling effect. Students in the deictic condition reported the highest retention score. Guiding attention on learning relevant parts of the videos and therefore, integrating the lecturer and the additional 
illustrative material seemed crucial for learning which supports results regarding the signaling effect (e.g., Boucheix, et al., 2013). Thus, recent results regarding the benefits of deictic gestures used by actual humans could be replicated (Pi et al., 2020). In contrast to beat gestures, deictic gestures are an important design feature that can foster learning in environments with humans, as well as digital agents (e.g., Li et al., 2019).

A further look into learning relevant self-report measures revealed that gestures were not able to increase the affective rating and therefore, enjoyment of the video. This might be an indication that the current results are mostly based on cognitive and social processes and not on affective variables. Furthermore, regarding the PSI sub-facets, only attention processes showed significant effects. Gestures did not seem to influence further parasocial processes because students were more focused on the lecture about the complex topic and distributed their attention between the lecturer and the additional illustrative materials. Therefore, additional parasocial processes like evaluation and anticipation of future behavior might not be triggered.

A crucial limitation of Experiment 1 is the implementation of the additional illustrative material in the deictic condition. As mentioned, in the deictic condition, illustrations were placed in a way that illustrations matched with the gestures of the lecturer. Therefore, the additional illustrative material differed in terms of size and distance to the lecturer. Although it was explicitly ensured that the additional illustrative information could be perceived easily in all conditions, the differing presentation of the illustrations might restrict internal validity. Therefore, a second experiment was carried out in order to eliminate this issue.

\section{Experiment 2}

Experiment 2 had two specific goals: (1) to replicate the findings from Experiment 1 to provide converging empirical evidence for the effectiveness of deictic gestures in educational videos and 
(2) to eliminate potential methodical issues, in particular, regarding the presentation of additional illustrative materials. Again, educational videos were manipulated in terms of the lecturer's gestures (no gestures versus beat gestures versus deictic gestures). Considering the results of Experiment 1, the following hypothesis and research questions were formulated for the second experiment:

H3: Learners who watch an educational video with a lecturer showing deictic beat gestures achieve higher learning scores than learners who watch an educational video with a lecturer showing no gestures.

RQ2: Do beat gestures influence learning outcomes?

RQ3: Do students who watch an educational video with a lecturer showing deictic gestures or beat gestures differ regarding learning scores?

Again, several self-report measures were investigated. In addition to those assessed in Experiment 1, the agent-persona perception was measured (Schroeder, Yang, Banerjee, Romine, \& Craig, 2018) to get deeper insights into the social learning process.

\subsection{Method}

Participants \& Design. For the second experiment, 121 students (75.2\% female; age: $M=$ 23.93, $S D=3.06$ ) from the XXX could be acquired. Again, participants were randomly assigned to one cell of a one-factorial between-participant design with three factor levels (no gestures versus beat gestures versus deictic gestures). Participants studied language studies (20\%), natural sciences (17.4\%), social sciences (17.4\%), medicine (7.8\%), computer sciences (4.3\%) and other fields of study (33\%). Thirty-seven students were assigned to the condition with no gestures, 43 students were assigned to the condition with rhythmic (beat) gestures and 41 students to the condition with signaling (deictic) gestures. There were no significant differences between the three treatment groups in terms of age, or prior knowledge, $F[2,118]=[1.27,2.71] ; p=[.07, .29]$ 
as well as gender, familiarity with the lecturer, or field of study $\chi^{2}=[1.13,17.72] ; p=[.06, .57]$. Again, no covariate had to be included in the analyses.

Materials. The materials were based on the materials of Experiment 1. A pre-trained male lecturer held an oral presentation on facts about industrial revolution. The lecture contained information about history and economic progress during the industrial revolution as well as social problems during this time. Again, the content was chosen because prior knowledge of participants was considered low and additional illustrations could be designed to provide further explanations in the video. Overall, the experimental videos lasted about 9:20 minutes.

Lecturer. The lecturer was presented in the right half of the screen (see Figure 3). Gestures were implemented in the same way as in experiment 1.

\section{Insert Figure 3 about here}

Additional illustrational material. Materials were implemented the same way as in Experiment 1. Illustrations were updated regularly while showing relevant technical progression, historical figures, geographical maps, and caricatures. Again, in the deictic condition, illustrations were placed in a way, that they matched with the gestures of the lecturer. In contrast to Experiment 1, placement of illustrations in the deictic condition was also used in the other conditions. As such, in Experiment 2, the additional illustrational material was kept constant across conditions. Examples of the material regarding the urbanization in Great Britain are displayed in Figure 4.

\section{Insert Figure 4 about here}

\section{Measures.}

Mental Load and Mental Effort. In line with Experiment 1, mental load $(\omega=.86)$ and mental effort ( $\omega=.77)$ were measured with the questionnaire developed by Krell (2015). In appendix A, all items of all scales are displayed. 
Affective Rating. Again, a scale from Linnenbrink-Garcia and colleagues $(2010 ; \omega=.95)$ was used.

Social Presence. Social presence $(\omega=.51)$ was measured on a scale from Bailenson, Aharoni, Beal, Guadagno, Dimov, and Blascovich (2004).

Parasocial Interaction. Again, the PSI process scales (Schramm \& Hartmann, 2008) were used. Eight items were chosen $(\omega=.62)$ which measured the same four sub-facets as in Experiment 1: accuracy of processing lecturer-related information, attention towards the persona, logical understanding of the behaviour of the persona, and evaluation of the persona.

Agent-persona perception. Agent-persona perception was measured with the agent persona instrument (Ryu \& Baylor, 2005) in its latest revision (API-R; Schroeder et al., 2018). Ten items measured whether the persona facilitated learning $(\omega=.91$; e.g., "The lecturer made the instruction interesting”). Five items measured whether the persona was credible $(\omega=.82$; e.g., “The agent was knowledgeable”) and five items measured whether the persona was engaging ( $\omega$ = .88; e.g., “The agent was enthusiastic”). Participants had to rate items on a 5-point Likert scale ranging from "strongly disagree” to "strongly agree”. The sub-scale "Human-like” was not used in the current experiment because the same real person was filmed in all conditions and anthropomorphic cues were not manipulated.

Prior Knowledge. Prior knowledge was assessed by five questions such as "In which country did the industrial revolution begin?” For the first two questions, students were awarded one point when they answered correctly. As the last three questions were more complex, students were awarded up to four points per question depending on the number of correct facts. In sum, students could achieve a maximum of 14 points on the prior knowledge test. Inter-rater reliability of two pre-trained reviewers was high; ICC $(1, \mathrm{k})=[.698, .989], F(120,120)=[5.62,174.75], p<.001$. 
Retention and Transfer. A comparable test as in Experiment 1 was used in order to measure retention $(\omega=.60)$ and transfer $(\omega=.47)$. Again, reliability of the knowledge measures is rather low because of the method of item construction (see Experiment 1). Again, the reliabilities were low. This can be explained considering the scale creation. Both constructs were measured as multidimensional constructs (see Experiment 1 for further details).

Retention was measured with 12 multiple choice questions (e.g., "When did the industrial revolution begin?”). Students were asked to choose between five solution probes with each question having up to five correct probes. Students received points for selecting correct answers and for not selecting incorrect answers. Students were thus able to get up to five points per question - and a maximum of 60 points in the retention test.

Transfer was measured with three multiple choice questions and four open questions (e.g., “What would have happened if Great Britain had no colonies?”). In sum, students could reach up to 18 points in the transfer test. With regard to the open questions, inter-rater reliability of two pre-trained reviewers was high; ICC $(1, \mathrm{k})=[.530, .771], F(120,120)=[3.25,7.74], p<.001$.

Procedure. The experiment lasted about 45 minutes and took place in a computer lab in XXX. The procedure was identical to Experiment 1.

\subsection{Results}

The same analysis-procedure as in Experiment 1 was used. Descriptive results for all dependent variables are displayed in Table 2.

\section{Insert Table 2 around here.}

Affective Rating. An ANOVA with affective rating as the dependent variable showed no significant effect for the between-participant factor gesture instruction; $F(2,118)=0.43, p=.65$. 
Social Presence. An ANOVA with social presence as dependent variable revealed a significant effect of gesture instruction with a medium effect size; $F(2,118)=3.75, p=.03, \eta_{\mathrm{p}}{ }^{2}=.06$. Sidakcorrected post-hoc analyses revealed that students in the deictic condition reported higher social presence than students in the control condition $\left(M_{\mathrm{diff}}=0.54 ; p=.03\right)$. None of the other pairwise comparisons were significant. Because of the low reliability of the social presence scale, this result must be interpreted with caution.

Parasocial Interaction. A MANOVA with PSI sub-facets (accuracy of persona-relevant information, attention, logical understanding, evaluation) as dependent variables revealed a significant effect of the factor gesture instruction with a medium effect size; Wilk's $\Lambda=0.78 ; F(8$, $230)=3.81, p<.001, \eta_{\mathrm{p}}^{2}=.12$. Univariate ANOVAs indicated a significant effect of gesture instruction with a medium to large effect size for attention towards the persona; $F(2,118)=8.27$, $p<.001, \eta_{\mathrm{p}}{ }^{2}=.12$. Sidak-corrected post-hoc analyses revealed that students in the deictic condition reported higher scores of attention than students in the control condition $\left(M_{\text {diff }}=0.81\right.$; $p=.01)$ and the beat condition $\left(M_{\mathrm{diff}}=1.03 ; p<.001\right)$. There also was a significant effect with a medium effect size for accuracy of persona-relevant information; $F(2,118)=4.65, p=.01, \eta_{\mathrm{p}}^{2}=$ .07. Sidak-corrected post-hoc analyses indicated that students in the deictic condition also reported higher accuracy than students in the control condition $\left(M_{\mathrm{diff}}=0.91 ; p=.01\right)$. Finally, there was a significant effect of gesture instruction with a small effect size for evaluation; $F(2$, 118) $=3.16, p=.046, \eta_{\mathrm{p}}^{2}=.05$. Sidak-corrected post hoc analyses showed no significant differences between groups. Nevertheless, there was a tendency indicating that students in the deictic condition reported higher evaluation scores than students in the beat condition $\left(M_{\text {diff }}=\right.$ $0.53 ; p=.08)$. There was no significant effect on logical understanding; $F(2,118)=1.56, p=.29$. 
Agent-persona perception. A MANOVA was conducted with API-R facets (learning facilitating, credibility, engagement) as dependent variables. A significant effect of gesture instruction with a medium effect size was found; Wilk's $\Lambda=0.87 ; F(4,232)=2.76, p=.01, \eta_{\mathrm{p}}{ }^{2}$ $=.07$. Univariate ANOVAs showed a significant effect of gesture instruction with a medium effect size for learning facilitating; $F(2,118)=6.41, p=.002, \eta_{\mathrm{p}}^{2}=.10$, credibility; $F(2,118)=3.25, p$ $=.04, \eta_{\mathrm{p}}{ }^{2}=.05$, and engagement; $F(2,118)=7.60, p=.001, \eta_{\mathrm{p}}{ }^{2}=.11$. Sidak-corrected post-hoc analyses indicated that the lecturer was perceived to be more beneficial for learning in the deictic condition in contrast to the control $\left(M_{\text {diff }}=0.64 ; p=.003\right)$ and the beat gesture condition $\left(M_{\text {diff }}=\right.$ $0.47 ; p=.03)$. Additionally, the lecturer was experienced to be more credible in the deictic condition in contrast to the control condition $\left(M_{\text {diff }}=0.46 ; p=.04\right)$ and rated more engaging in the deictic condition in contrast to the control $\left(M_{\text {diff }}=0.72 ; p=.001\right)$ and the beat condition $\left(M_{\text {diff }}\right.$ $=0.58 ; p=.01)$

Mental Load and Mental Effort. A MANOVA was conducted with mental load (ML) and mental effort (ME) as dependent variables. A significant effect of gesture instruction with small to medium effect size was observed; Wilk's $\Lambda=0.90 ; F(4,234)=3.08, p=.02, \eta_{\mathrm{p}}^{2}=.05$. Univariate ANOVAs revealed a significant effect of gesture instruction for $\mathrm{ME} ; F(2,118)=4.59$, $p=.01, \eta_{\mathrm{p}}^{2}=.07$, but no significant effect for $\mathrm{ML} ; F(2,118)=2.18, p=.12$. Regarding ME, Sidak-corrected post-hoc analyses indicated that students in the deictic condition reported lower ME than students in the control condition $\left(M_{\mathrm{diff}}=0.58 ; p=.01\right)$.

\section{Learning Outcomes.}

A MANOVA was conducted with retention and transfer scores as dependent variables. There was a significant main effect of gesture instruction with a medium effect size; Wilk's $\Lambda=0.88$; $F(4,234)=4.01, p=.004, \eta_{\mathrm{p}}{ }^{2}=.07$. Univariate ANOVAs revealed a significant effect of gesture 
instruction with a medium effect size for retention; $F(2,118)=4.96, p=.01, \eta_{\mathrm{p}}{ }^{2}=.08$. Sidakcorrected post-hoc analyses revealed that students in the deictic condition reported higher retention scores than students in the control $\left(M_{\mathrm{diff}}=3.74 ; p=.01\right)$ and in the beat gesture condition $\left(M_{\text {diff }}=3.15 ; p=.04\right)$. A significant effect of gesture instruction with a medium effect size was also observed for transfer; $F(2,118)=4.79, p=.01, \eta_{\mathrm{p}}{ }^{2}=.08$. Sidak-corrected post-hoc analyses indicated that students in the deictic condition reported higher retention scores than students in the control condition $\left(M_{\mathrm{diff}}=1.53 ; p=.01\right)$ but not the beat condition $\left(M_{\mathrm{diff}}=1.11 ; p=.09\right)$.

\subsection{Discussion}

In line with the results of Experiment 1, results of Experiment 2 demonstrated that deictic gestures enhanced learning outcomes. Therefore, H3 was supported by our data. Regarding RQ2, it was again observed that beat gestures did not significantly foster learning outcomes. With respect to RQ3, the current results indicate that the deictic condition outperformed the beat condition in terms of retention, but not transfer.

In sum, these results support the assumptions postulated from Experiment 1. Implementation of deictic gestures in educational videos significantly enhanced social presence and attention towards the lecturer. Furthermore, lecturers who performed deictic gestures were perceived to foster learning, to be more credible and engaging. This indicates that the attention of the learner was successfully drawn to important illustrative information by gestures as non-verbal behavior from a human lecturer. This substantiates recent findings from Pi and colleagues (2020). Because the lecturer interacts with the additional illustrative material through deictic gestures, both lecturer and illustrative material are always in the center of the learners' attention and - in line with parasocial theories - social information was processed more deeply (Hartmann et al., 2004, Klimmt et al., 2006). Additionally, split attention was seemingly lowered and consequently, the 
necessary mental effort as well. Again, these findings supported results regarding the benefits of deictic gestures (Li et al., 2019) and extended them to human lecturers in instructional videos. Learning was fostered when the lecturer pointed directly at the information at the time the information was relevant for schema construction (Twyford \& Craig, 2013; Craig et al., 2015).

Importantly, current results indicated that beat gestures did not foster learning. Thus, the embodiment principle (Mayer, 2014) was not supported without boundary conditions. As such, social cues, which are implemented in an educational video without relevance to the actual learning content, did neither foster nor inhibit learning processes. In particular, when additional illustrative material was shown within an instructional video, learners may have split their attention between lecturer and illustrative material. Consequently, social cues that do not interact with the material may not have relevant influences in social perception and cognitive processing of the learner. In contrast to instructional videos in which only the lecturer is in the main focus of the video (e.g., Beege et al., 2017), social cues may have to be implemented in line with well based instructional design recommendations like the signaling principle (e.g., Schneider et al., 2018).

Importantly, results regarding certain PSI facets and ME differed between the two experiments. This might be explained with the change of the arrangement of the additional illustrative material. Nevertheless, the vast majority of results was replicated. This indicated that the interaction between the lecturer and illustrative material might be more important for learning than the basic arrangement of the material. Thus, directing the eye gaze and attention of learners on relevant visual material should be taken into account inevitably. Nevertheless, future research is necessary to evaluate this assumption. 


\section{Implications and Limitations}

On the theoretical side, the current results indicate that social cues in educational videos should be implemented carefully. When the lecturer is not the only focus of the video, social cues might not be relevant for learning because learners have to split their attention between lecturer and additional illustrative material. Thus, the lecturer is only partially in the focus of learners' attention and the embodiment principle or effects of anthropomorphism cannot unfold. Social cues should be implemented in a way to create illustrative associations within the learning material. When the lecturer and other sources of information are integrated through gestures of social entity, advantages of parasocial processes become apparent. Therefore, the signaling principle was supported by our data, which is another main implication of this study. The third implication outlines that gestures from actual human lecturers seem to be processed identically to gestures performed by virtual agents. Li and colleagues (2019) investigated virtual agents and their results regarding learning were replicated in the current investigation. This supports the CASA paradigm (Nass et al., 1994). Basic social cues prime a social activation schema and in consequence, social processes of human-to-human communication. A digital, as well as a human lecturer, can use deictic gestures efficiently to draw the attention of learners to relevant information and to strengthen social processes. Finally, results about the subjective measurements made the underlying processes visible which complements previous studies. Performing deictic gestures is an effective way to optimize the attention allocation of the learner. Furthermore, learners are engaged to evaluate the persona in more detail and thus, learners had subjective accuracy in remembering persona-related variables. The video-lecturer is perceived as more credible, engaging, and facilitates learning when performing deictic gestures. Social presence is 
strengthened which enhanced processes of (para-)social communication processes. In consequence of all these changes, learning is fostered.

From these implications, practical design recommendations can be derived. Designers should be encouraged to create educational videos with signaling gestures in order to foster learning processes. In particular, when additional illustrative material is presented in the video, designers should consider how relevant parts of the video could be highlighted through the lecturer. Gestures beyond signaling do not seem necessary in order encourage learning processes. This was observed in the current study for a natural science topic as well as a historical topic indicating that using deictic gestures might be suitable across different learning contents.

Because of the experimental design of the video, three separate videos were filmed successively. Although speed of speech was kept constant and other confounding was minimized, minimal changes of facial expressions between experimental conditions could not be avoided. Only short-term effects of educational videos on learning with multimedia can be interpreted and discussed. This study cannot answer the question of whether the observed learning effects may be stable over a longer period of time. Finally, the low reliability of the social presence scale should be taken into account and the results should be interpreted with caution.

\section{Future Directions}

The effects of social cues in multimedia learning are not thoroughly investigated yet. There is much to do for future research regarding gestures, facial expressions, and other non-verbal or verbal cues, which can be used by lecturers in educational videos. Amongst others, future research should focus on the role of gestures when no additional illustrative material is provided in the video. It is possible that rhythmic gestures foster learning when the learners' focus of attention is always on the lecturer. Furthermore, signaling gestures can be implemented in videos without 
additional illustrative material as well. Certain gestures can underline important parts of speech or clarify relationships. Thus, signaling might be more than just guiding visual attention through deictic movements.

\section{References}

Amadieu, F., Mariné, C., \& Laimay, C. (2011). The attention-guiding effect and cognitive load in the comprehension of animations. Computers in Human Behavior, 27, 36-40.

Atkinson, R. K. (2002). Optimizing learning from examples using animated pedagogical agents. Journal of Educational Psychology, 94, 416-427.

Bailenson, J. N., Aharoni, E., Beall, A. C., Guadagno, R. E., Dimov, A., \& Blascovich, J. (2004, October). Comparing behavioral and self-report measures of embodied agents’ social presence in immersive virtual environments. In Proceedings of the 7th Annual International Workshop on PRESENCE (pp. 1864-1105).

Barsalou, L. W. (2010). Grounded cognition: Past, present, and future. Topics in Cognitive Science, 2, 716-724.

Baylor, A. L., \& Kim, S. (2009). Designing nonverbal communication for pedagogical agents: When less is more. Computers in Human Behavior, 25, 450-457.

Beege, M., Schneider, S., Nebel, S., \& Rey, G. D. (2017). Look into my eyes! Exploring the effect of addressing in educational videos. Learning and Instruction, 49, 113-120.

Blascovich, J., \& Bailenson, J. (2011). Infinite reality: Avatars, eternal life, new worlds, and the dawn of the virtual revolution. New York: William Morrow \& Co.

Boucheix, J. M., Lowe, R. K., Putri, D. K., \& Groff, J. (2013). Cueing animations: Dynamic signaling aids information extraction and comprehension. Learning and Instruction, 25, 71-84. 
Brooks, N., \& Goldin-Meadow, S. (2016). Moving to learn: How guiding the hands can set the stage for learning. Cognitive Science, 40, 1831-1849.

Cassell, J., \& McNeill, D. (1991). Gesture and the poetics of prose. Poetics Today, 12, 375-404.

Cassell, J., McNeill, D., \& McCullough, K. E. (1999). Speech-gesture mismatches: Evidence for one underlying representation of linguistic and nonlinguistic information. Pragmatics \& Cognition, 7, 1-34.

Congdon, E. L., Novack, M. A., Brooks, N., Hemani-Lopez, N., O'Keefe, L., \& Goldin-Meadow, S. (2017). Better together: Simultaneous presentation of speech and gesture in math instruction supports generalization and retention. Learning and Instruction, 50, 65-74.

Cook, S. W., Duffy, R. G., \& Fenn, K. M. (2013). Consolidation and transfer of learning after observing hand gesture. Child Development, 84, 1863-1871.

Cook, S. W., Friedman, H. S., Duggan, K. A., Cui, J., \& Popescu, V. (2017). Hand gesture and mathematics learning: lessons from an Avatar. Cognitive Science, 41, 518-535.

Cook, S.W., Mitchell, Z., \& Goldin-Meadow, S. (2008). Gesturing makes learning last. Cognition, 106, 1047-1058.

Craig, S. D., Gholson, B., \& Driscoll, D. (2002). Animated pedagogical agents in multimedia educational environments: Effects of agent properties, picture features, and redundancy. Journal of Educational Psychology, 94, 428-434.

Craig, S. D., Twyford, J., Irigoyen, N., \& Zipp S. (2015). A test of spatial contiguity for virtual human's gestures in multimedia learning environments. Journal of Educational Computing Research, 53, 3-14.

Davis, R. O. (2018). The impact of pedagogical agent gesturing in multimedia learning environments: A meta-analysis. Educational Research Review, 24, 193-209. 
Davis, R. O., \& Vincent, J. (2019). Sometimes more is better: Agent gestures, procedural knowledge and the foreign language learner. British Journal of Educational Technology, 50, 3252-3263.

Dunsworth, Q., \& Atkinson, R. K. (2007). Fostering multimedia learning of science: Exploring the role of an animated agent's image. Computers \& Education, 49, 677-690.

Faul, F., Erdfelder, E., Lang, A.-G., \& Buchner, A. (2007). G*Power 3: A flexible statistical power analysis program for the social, behavioral, and biomedical sciences. Behavior Research Methods, 39, 175-191.

Ginns, P. (2006). Integrating information: A meta-analysis of the spatial contiguity and temporal contiguity effects. Learning and Instruction, 16, 511-525.

Glenberg, A. M. (2010). Embodiment as a unifying perspective for psychology. Wiley Interdisciplinary Reviews: Cognitive Science, 1, 586-596.

Gola, A. A., Richards, M. N., Lauricella, A. R., \& Calvert, S. L. (2013). Building meaningful relationships between toddlers and media characters to teach early mathematical skills. Media Psychology, 16, 390-411.

Goldin-Meadow, S. (2011). Learning through gesture. Wiley Interdisciplinary Reviews. Cognitive Science, 2, 595-607.

Grice, H. P. (1975). Logic and conversation. In P. Cole \& J. Morgan (Eds.), Syntax and Semantics (Vol. 3, pp. 41-58). New York: Academic Press.

Harp, S. F., \& Mayer, R. E. (1998). How seductive details do their damage: A theory of cognitive interest in science learning. Journal of Educational Psychology, 90, 414-434.

Hartmann, T., Schramm, H. \& Klimmt, C. (2004). Personenorientierte Medienrezeption: Ein Zwei-Ebenen-Modell parasozialer Interaktionen. Publizistik, 49, 25-47. 
Horton, D., \& Wohl, R. R. (1956). Mass communication and para-social interaction: Observation on intimacy at a distance. Psychiatry, 19, 185-206.

Kalyuga, S., \& Singh, A. M. (2016). Rethinking the boundaries of cognitive load theory in complex learning. Educational Psychology Review, 28, 831-852.

Kizilcec, R. F., Bailenson, J. N., \& Gomez, C. J. (2015). The instructor’s face in video instruction: Evidence from two large-scale field studies. Journal of Educational Psychology, 107, 724-739.

Klimmt, C., Hartmann, T., \& Schramm, H. (2006). Parasocial interactions and relationships. In J. Bryant \& P. Vorderer (Eds.), Psychology of entertainment (pp. 291-313). Mahwah, NJ: Erlbaum.

de Koning, B. B., \& Tabbers, H. K. (2013). Gestures in instructional animations: A helping hand to understanding non-human movements?. Applied Cognitive Psychology, 27, 683-689.

Koumoutsakis, T., Church, R. B., Alibali, M. W., Singer, M., \& Ayman-Nolley, S. (2016). Gesture in instruction: Evidence from live and video lessons. Journal of Nonverbal Behavior, 40, 301-315.

Krell, M. (2015). Evaluating an instrument to measure mental load and mental effort using item response theory. Science Education Review Letters, 1-6.

Lee, K. M., \& Nass, C. (2003, April). Designing social presence of social actors in human computer interaction. In Proceedings of the SIGCHI conference on Human factors in computing systems (pp. 289-296). ACM.

Li, W., Wang, F., Mayer, R. E., \& Liu, H. (2019). Getting the point: Which kinds of gestures by pedagogical agents improve multimedia learning? Journal of Educational Psychology. Advance online publication. 
Lin, L., \& Atkinson, R. K. (2011). Using animations and visual cueing to support learning of scientific concepts and processes. Computers \& Education, 56, 650-658.

Linnenbrink-Garcia, L., Durik, A. M., Conley, A. M., Barron, K. E., Tauer, J. M., Karabenick, S. A., \& Harackiewicz, J. M. (2010). Measuring situational interest in academic domains. Educational and Psychological Measurement, 70, 647-671.

Luo, H., Koszalka, T., \& Zuo, M. (2016). Investigating the effects of visual cues in multimedia instruction using eye tracking. In S. K. S. Cheung, L. Kwok, J. Shang, A. Wang, R. Kwan (Eds.), Blended Learning: Aligning Theory with Practices, (pp. 63-72). Springer International Publishing.

Mayer, R. E. (2001). Multimedia learning. New York: Cambridge University Press.

Mayer, R. E. (2014). The Cambridge handbook of multimedia learning (2 ed.). Cambridge, MA: Cambridge University Press.

Mayer, R. E., \& Estrella, G. (2014). Benefits of emotional design in multimedia instruction. Learning and Instruction, 33, 12-18.

Mayer, R. E. \& Moreno, R. (2003). Nine ways to reduce cognitive load in multimedia learning. Educational Psychologist, 38, 43-52.

McDonald, R. P. (1999). Test theory: A unified treatment. Mahwah, NJ: Lawrence Erlbaum Associates.

McNeill, D. (1992). Hand and mind: What gestures reveal about thought. Chicago: The University of Chicago press.

McNeish, D. (2017, May 29). Thanks Coefficient Alpha, We’ll Take It From Here. Psychological Methods. Advance online publication. 
Nass, C., Steuer, J., \& Tauber, E. R. (1994, April). Computers are social actors. In Proceedings of the SIGCHI conference on Human factors in computing systems (pp. 72-78). ACM.

Novack, M., \& Goldin-Meadow, S. (2015). Learning from gesture: how our hands change our minds. Educational Psychology Review, 27, 405-412.

Ouwehand, K., Van Gog, T., \& Paas, F. (2015). Effects of gestures on older adults' learning from video-based models. Applied Cognitive Psychology, 29, 115-128.

Paas, F., \& Sweller, J. (2014). Implications of cognitive load theory for multimedia learning. In R. E. Mayer (Ed.), The Cambridge handbook of multimedia learning (2nd ed., pp. 27-42). Cambridge: Cambridge University Press.

Park, S. (2015). The effects of social cue principles on cognitive load, situational interest, motivation, and achievement in pedagogical agent multimedia learning. Journal of Educational Technology \& Society, 18, 211.

Pi, Z., Xu, K., Liu, C., \& Yang, J. (2020). Instructor presence in video lectures: Eye gaze matters, but not body orientation. Computers \& Education, 144, 103713.

Ping, R., Goldin-Meadow, S., \& Beilock, S. (2014). Understanding gesture: Is the listener's motor system involved. Journal of Experimental Psychology General, 143, 195-204.

Plass, J. L., Heidig, S., Hayward, E. O., Homer, B. D., \& Um, E. (2014). Emotional design in multimedia learning: Effects of shape and color on affect and learning. Learning and Instruction, 29, 128-140.

Reeves, B., \& Nass, C. (1996). The media equation. New York: Cambridge University Press.

Roth, W. M. (2001). Gestures: Their role in teaching and learning. Review of Educational Research, 71, 365-392. 
Ryu, J., \& Baylor, A. L. (2005). The Psychometric Structure of Pedagogical Agent Persona. Technology Instruction Cognition and Learning, 2, 291-315.

Salem, M., Kopp, S., Wachsmuth, I., Rohlfing, K., \& Joublin, F. (2012). Generation and evaluation of communicative robot gesture. International Journal of Social Robotics, 4, 201217.

Schneider, S., Beege, M., Nebel, S., \& Rey, G. D. (2018a). A meta-analysis of how signaling affects learning with media. Educational Research Review, 23, 1-24.

Schneider, S., Nebel, S., Beege, M. \& Rey, G. D. (2018b). Anthropomorphism in decorative illustrations: Benefit or harm for learning? Journal of Educational Psychology, 110, 218-232.

Schramm, H., \& Hartmann, T. (2008). The PSI-Process Scales. A new measure to assess the intensity and breadth of parasocial processes. Communications, 33, 385-401.

Schroeder, N. L., \& Cenkci, A. T. (2018). Spatial Contiguity and Spatial Split-Attention Effects in Multimedia Learning Environments: a Meta-Analysis. Educational Psychology Review. Advanced Online Publication.

Schroeder, N. L., Yang, F., Banerjee, T., Romine, W. L., \& Craig, S. D. (2018). The influence of learners' perceptions of virtual humans on learning transfer. Computers \& Education, 126, 170182.

Shrout, P. E., \& Fleiss, J. L. (1979). Intraclass correlations: uses in assessing rater reliability. Psychological Bulletin, 86, 420.

Stieff, M., Lira, M. E., \& Scopelitis, S. A. (2016). Gesture supports spatial thinking in STEM. Cognition and Instruction, 34, 80-99.

Sundararajan, N., \& Adesope, O. (2020). Keep it Coherent: A Meta-Analysis of the Seductive Details Effect. Educational Psychology Review. Advanced Online Publication. 
Sweller, J. (1988). Cognitive load during problem solving: Effects on learning. Cognitive Science, 12, 257-285.

Twyford, J., \& Craig, S. D. (2013). Virtual humans and gesturing during multimedia learning: An investigation of predictions from the temporal contiguity effect. In T. Bastiaens \& G. Marks (Eds.), World Conference on E-Learning in Corporate, Government, Healthcare, and Higher Education (ELEARN) 2013 (pp. 2541-2545). Chesapeake, VA: AACE.

Um, E., Plass, J. L., Hayward, E. O., \& Homer, B. D. (2012). Emotional design in multimedia learning. Journal of Educational Psychology, 104, 485-498.

Wakefield, E., Novack, M. A., Congdon, E. L., Franconeri, S., \& Goldin-Meadow, S. (2018). Gesture helps learners learn, but not merely by guiding their visual attention. Developmental Science, e12664.

Wang, F., Li, W., Mayer, R. E., \& Liu, H. (2018). Animated pedagogical agents as aids in multimedia learning: Effects on eye-fixations during learning and learning outcomes. Journal of Educational Psychology, 110, 250-268. 




Condition without gestures

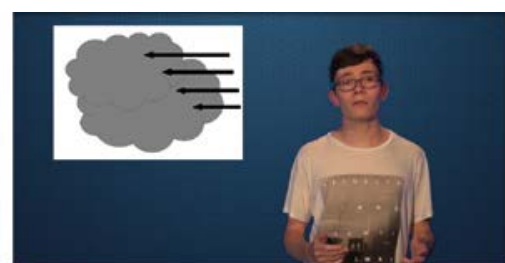

Condition with beat gestures

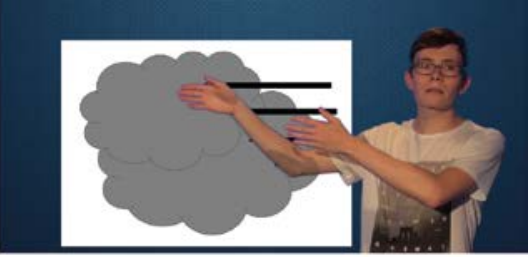

Condition with deictic gestures

Figure 1. Illustration of the experimental manipulation of Experiment 1. 


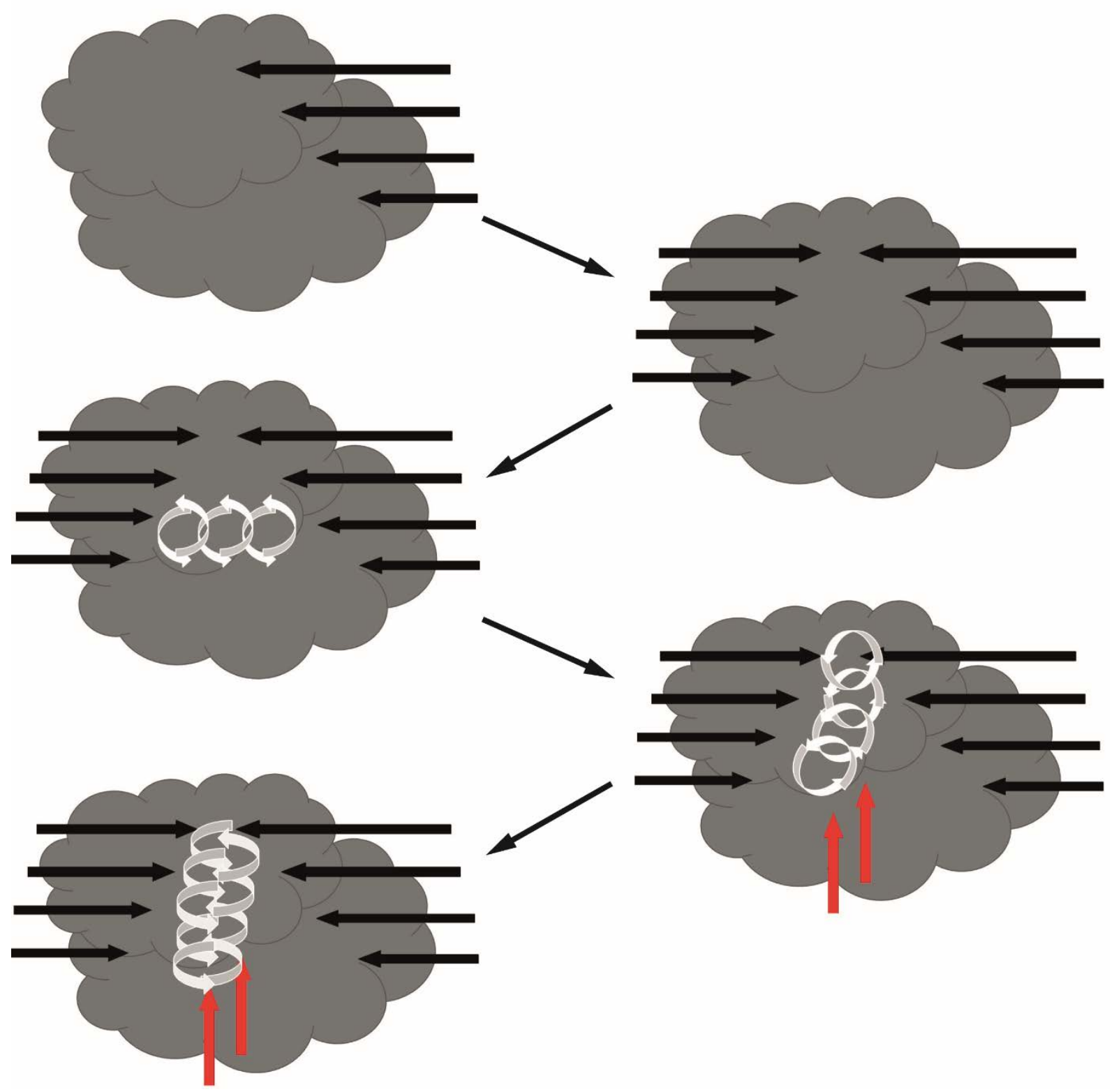

Figure 2. Example of the additional pictorial material of Experiment 1 for the sub-topic tornados. 




Condition without gestures

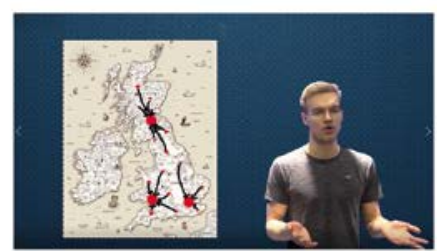

Condition with beat gestures



Condition with deictic gestures

Figure 1. Illustration of the experimental manipulation of Experiment 2. 


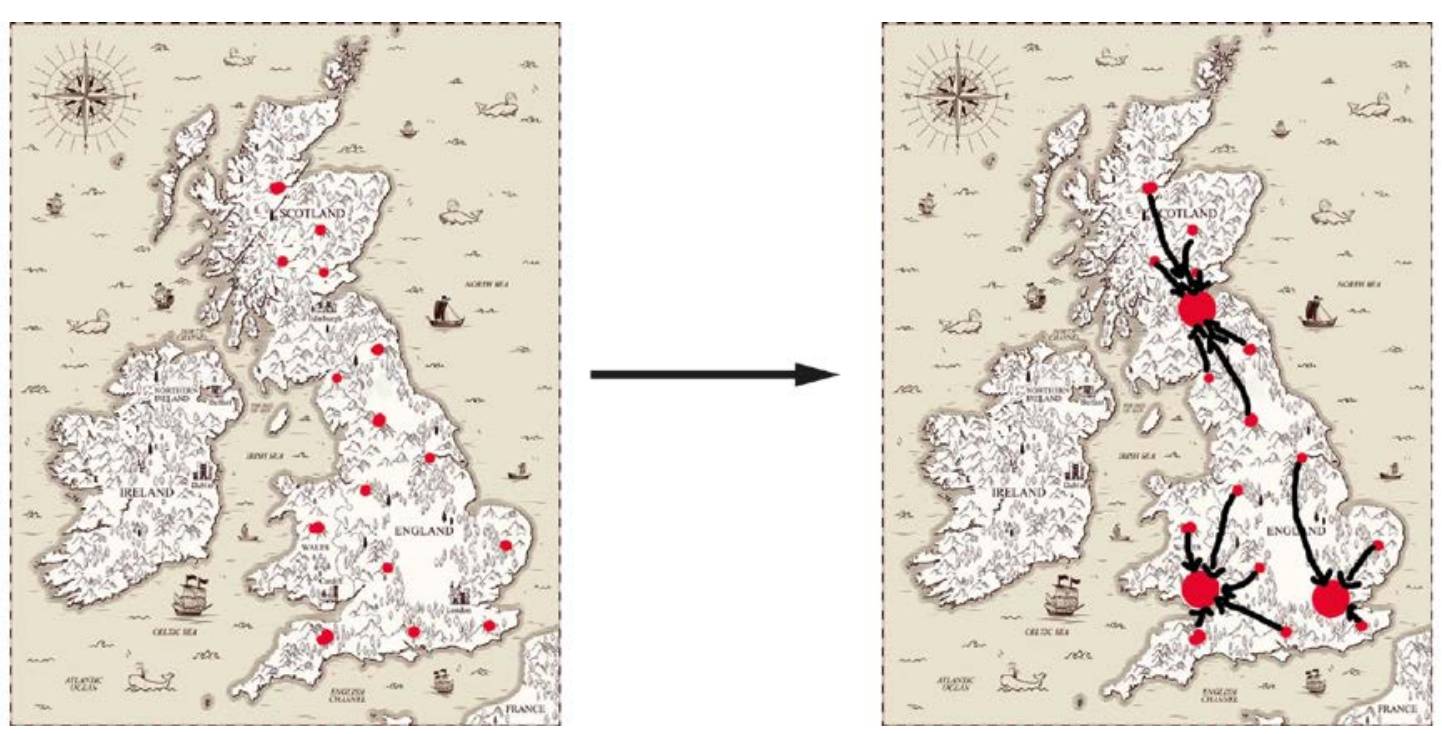

Figure 4. Example of the additional pictorial material of Experiment 2 for the sub-topic urbanization in Great Britain. 
Table 1. Mean and standard deviations of all dependent variables for the three experimental groups of Experiment 1.

\begin{tabular}{lcccccc}
\hline & \multicolumn{5}{c}{ Experimental groups } \\
\cline { 2 - 6 } & \multicolumn{2}{c}{$\begin{array}{c}\text { Signaling Gestures } \\
(N=39)\end{array}$} & $\begin{array}{c}\text { Rhythmic Gestures } \\
(N=33)\end{array}$ & $\begin{array}{c}\text { No Gestures } \\
(N=36)\end{array}$ \\
\hline \multirow{2}{*}{$\begin{array}{l}\text { Affective Rating } \\
\text { Social Presence }\end{array}$} & 4.15 & 1.59 & 3.76 & 1.36 & 3.54 & 1.61 \\
Perceptive-Cognitive PSI & 2.94 & 0.76 & 2.68 & 0.69 & 2.32 & 0.77 \\
$\quad$ Accuracy of Processing Information & 5.67 & 1.47 & 5.45 & 1.50 & 5.64 & 1.44 \\
$\quad$ Attention towards the Persona & 5.35 & 1.12 & 4.76 & 1.64 & 4.00 & 1.51 \\
$\quad$ Logical Understanding of the Behavior & 4.32 & 1.20 & 4.27 & 1.09 & 4.04 & 1.45 \\
$\quad$ Evaluation of the Persona & 4.08 & 1.06 & 4.12 & 1.39 & 3.69 & 1.31 \\
ML & 4.02 & 1.08 & 4.35 & 1.17 & 4.50 & 1.10 \\
ME & 5.35 & 0.61 & 4.96 & 0.87 & 5.39 & 0.65 \\
Retention & 12.03 & 1.58 & 10.76 & 2.12 & 10.69 & 2.05 \\
Transfer & 3.67 & 1.22 & 3.15 & 1.11 & 3.26 & 1.10 \\
\hline
\end{tabular}

Note. PSI = Parasocial Interaction, ML = Mental Load, ME = Mental Effort, $M=$ mean scores, $S D=$ standard deviation. 
Table 2. Mean and standard deviations of all dependent variables for the three experimental groups of Experiment 2.

\begin{tabular}{|c|c|c|c|c|c|c|}
\hline & \multicolumn{6}{|c|}{ Experimental groups } \\
\hline & \multicolumn{2}{|c|}{$\begin{array}{l}\text { Signaling Gestures } \\
\qquad(N=37)\end{array}$} & \multicolumn{2}{|c|}{$\begin{array}{l}\text { Rhythmic Gestures } \\
\qquad(N=43)\end{array}$} & \multicolumn{2}{|c|}{$\begin{array}{l}\text { No Gestures } \\
\quad(N=41)\end{array}$} \\
\hline & $M$ & $S D$ & $M$ & $S D$ & $M$ & $S D$ \\
\hline Affective Rating & 3.85 & 1.70 & 3.68 & 2.50 & 3.53 & 1.51 \\
\hline Social Presence & 3.72 & 0.78 & 3.32 & 1.00 & 3.17 & 0.96 \\
\hline \multicolumn{7}{|l|}{ Perceptive-Cognitive PSI } \\
\hline Accuracy of Processing Information & 3.88 & 1.35 & 3.47 & 1.39 & 2.97 & 1.17 \\
\hline Attention towards the Persona & 5.00 & 1.24 & 3.97 & 1.19 & 4.19 & 1.22 \\
\hline Logical Understanding of the Behavior & 4.48 & 1.29 & 4.03 & 1.32 & 4.30 & 1.22 \\
\hline Evaluation of the Persona & 4.92 & 0.99 & 4.39 & 1.18 & 4.14 & 1.04 \\
\hline \multicolumn{7}{|l|}{ Agent-Persona perception } \\
\hline Learning fostering & 3.28 & 0.80 & 2.81 & 0.84 & 2.64 & 0.83 \\
\hline Credibility & 3.58 & 0.82 & 3.31 & 0.86 & 3.12 & 0.69 \\
\hline Engaging & 2.93 & 0.84 & 2.35 & 0.88 & 2.21 & 0.90 \\
\hline ML & 3.54 & 0.96 & 3.99 & 1.09 & 3.84 & 0.92 \\
\hline ME & 4.63 & 0.98 & 5.04 & 0.95 & 5.20 & 0.62 \\
\hline Retention & 45.12 & 5.42 & 41.98 & 6.10 & 41.38 & 5.58 \\
\hline Transfer & 9.51 & 2.20 & 9.09 & 2.22 & 7.99 & 2.29 \\
\hline
\end{tabular}

Note. PSI = Parasocial Interaction, ML = Mental Load, ME = Mental Effort, $M=$ mean scores, $S D=$ standard deviation 
Appendix A. Scales and items from both experiments

\section{Both experiments}

Scale Items

Affective Rating

I enjoyed watching the video.

I liked the video.

I had fun watching the video.

It was exciting to watch the video.

Social Presence

I perceived that I was in the presence of another person in the virtual learning environment.

I felt that the lecturer was watching me and was aware of my presence.

I often thought that the lecturer was not a real person.

The lecturer appeared to be sentient, conscious and alive to me.

The lecturer was only a computerized image and not a real person.

Parasocial Interaction I still can remember what the lecturer said. (accuracy)

The lecturer kept drawing my full attention. (attention)

I hardly paid any attention to the lecturer. (attention)

I hardly thought about why the lecturer said certain things.

(logical understanding) 
I thought hard about what the speaker said. (logical

understanding)

I have always assessed whether I found the lecturer's statements

bad or good. (evaluation)

I noticed characteristics of the lecturer that I found good or bad.

(evaluation)

I have not formed an opinion about the things the lecturer said in the video. (evaluation)

Mental Load

The tasks were difficult to answer.

The contents of the tasks were complicated.

The tasks were challenging.

The tasks were easy to work on.

The contents of the tasks were easy to understand.

The tasks were easy to solve.

Mental Effort

At the processing of the tasks I haven’t done my best

particularly.

I haven't taken particular trouble with the reply to the tasks.

I have made an effort at the processing of the tasks.

At the reply to the tasks I have made an effort intellectually.

I haven’t particularly focused me to solve the tasks.

I have given my best to complete the tasks.

\section{Experiment 1}

Scale Items


What is the name of the trade wind in the northern hemisphere?

What is the name of the trade wind in the southern hemisphere?

Where do the two trade winds meet?

What is the high pressure area around the 30th platitude called?

What are the two types of shear?

What is the name of the vortex that does not touch the ground?

What is the name of the region in the United States where the most tornados occur?

On what scale is the strength of tornadoes measured?

What is the difference between tornados and hurricanes?

When do hurricanes occur especially often?

What is the minimum surface temperature of the water for hurricanes to occur?

What force causes air masses to rotate?

At what latitude do hurricanes occur?

Why are there no tornados around the equator?

One speaks of hurricanes from a wind speed of...

How many categories are hurricanes divided into?

Transfer

Why do tornados not arise at the arctic regions?

Hurricanes mostly occur during summer and autumn. Why is this the case? 
A sufficiently large sea area is a prerequisite for the formation of tornadoes. Why is there no hurricane in the North Sea despite fulfilling this requirement?

When do tropical whirlwinds occur in the waters around

Australia?

The course of tornado "Vardah" is shown in the following world map. Which designation applies to this tornado?

The migration of the zenith of the sun shifts the inner-tropical convergence zone and the passage circulation. Why does this make the Mediterranean climate warm and dry in summer and cold and humid in winter?

What is the correct order of the following pictures to describe the correct passage of the passage circulation?

Enter the appropriate name for the respective number in the following picture.

\section{Experiment 2}

Scale Items

Retention

When and where did the industrial revolution begin?

which specific circumstances favor industrialization in Great Britain?

Which agricultural conditions favor industrialization?

How did the industrial revolution begin?

Which 3 processes are summarized by Davis S. Landes to the core processes of industrialization? 
Which statements about the steam engine are correct?

Who was a pioneer in iron smelting in the coke oven?

Which statements on the social question are correct?

What is not a consequence of industrialization?

Why did industrialization start late in Germany?

Where did industrialization begin in Germany?

How did industrialization go in Germany?

Transfer

Why was Europe and not America the cradle of industrialization?

How would industrialization have been without colonies?

What are common rights in the context of industrialization?

Why was the industrial revolution not limited to one nation, but gradually appeared internationally?

Why wasn't the social question clarified and the symptoms

eliminated at the beginning?

In your opinion, what are the long-term political effects of the social question?

Why did European nations quickly catch up with Britain as a pioneer of industrialization?

API_R

Facilitating Learning
The lecturer led me to think more deeply about the presentation. The lecturer made the instruction interesting. The lecturer encouraged me to reflect what I was learning. The lecturer kept my attention. 
The lecturer communicated the main ideas clearly.

The lecturer helped me to concentrate on the presentation.

The lecturer focused me on the relevant information.

The lecturer helped me learn the material.

The lecturer was good at teaching.

The lecturer was easy to learn from.

API-R

The lecturer was knowledgeable.

Credible

The lecturer was intelligent.

The lecturer was useful.

The lecturer was helpful.

The lecturer was an effective teacher.

API-R

The lecturer was engaging.

Engaging

The lecturer was enthusiastic.

The lecturer was entertaining.

The lecturer was motivating.

The lecturer was easy to connect with. 